\title{
DIAGONALIZATION OF INCLUSIVE SUM RULES
}

$\triangle \mathrm{ND}$

\author{
INCLUSIVE BOOTSTRAP *
}

\author{
1 \\ by \\ Se-Yuen Mak and Chung-I Tan \\ Department of Physics \\ Brown University \\ Providence, Rhode Island 02912
}

This report was prepared as an account of work sponsored by the United States Government. Neither the United States nor the United States Atomic Energy Commission, nor any of their employees, nor any of their contractors, 'subcontractors, of their employees, makes any warranty, express or implied, or assumes any legal liability or responsibility for the accuracy, completeness or usefulness of any information, apparatus, product or process disclosed, or represents that its use would not infringe privately owned rights.

\begin{abstract}
We introduce the technique of multi-dimensional Laplace transforms under which the inclusive-like sum rules for discontinuities of multiparticle amplitudes can be diagonalized. The resulting equations are simple constraints on our generalized partial-wave amplitudes, and they can be used to impose unitarity conditions for bootstrap models. A simple example is given to illustrate the non-linear nature of this "inclusive bootstrap" program.

* Supported in part by the U.S. Atomic Energy Commission. (Report No. COO-3130TA-259).
\end{abstract}




\section{DISCLAIMER}

This report was prepared as an account of work sponsored by an agency of the United States Government. Neither the United States Government nor any agency Thereof, nor any of their employees, makes any warranty, express or implied, or assumes any legal liability or responsibility for the accuracy, completeness, or usefulness of any information, apparatus, product, or process disclosed, or represents that its use would not infringe privately owned rights. Reference herein to any specific commercial product, process, or service by trade name, trademark, manufacturer, or otherwise does not necessarily constitute or imply its endorsement, recommendation, or favoring by the United States Government or any agency thereof. The views and opinions of authors expressed herein do not necessarily state or reflect those of the United States Government or any agency thereof. 


\section{DISCLAIMER}

Portions of this document may be illegible in electronic image products. Images are produced from the best available original document. 


\section{Introduction}

The equivalence between the inclusive-like sum rules for discontinuities of multiparticle amplitudes ${ }^{1-4}$ and the basic discontinuity equations of Cahill and Stapp ${ }^{5}$ has renewed the hope of a practical enforcement of unitarity constraints at high energies. We have already shown that unitary models can be directly constructed from these linear sum rules under certain factorization approximations thus illustrating their potential usefulness as a new starting point for strong interaction dynamics: 6 Venezlano has noted that, in any approximate bootstrap scheme, these sum rules are non-linear relations in the "coupling constants", leading to the possibility of an "inclusive bootstrap". 1

We introduce a technique of handling these sum rules so that their non-linear characteristics can easily be exploited. Using multi-dimensional Laplace transforms we show that inclusive sum rules can be "diagonalized" in a set of "generalized" complex angular momenta. They then become exact constraints on "partial-wave" amplitudes of multiparticle discontinuities. Being statements of unitarity, they can be used: as a starting point for dynamical approximations.

Throughout this discussion, we assume that there is only one type of scalar particle, and we avoid the kinematic complications of transverse momenta by discussing only the integrated inclusive cross sections.' We use the normalization that, for $a+b \rightarrow 1+2+3 \cdots+n+$ anything; the discontinuity $D_{n}\left(p_{a}, p_{b} ; p_{1}, p_{2}, \cdots p_{n}\right)$ is related to the cross section by

$$
\frac{d \sigma}{d y_{1} d y_{2} \cdots d_{n}}=\Delta^{-\frac{1}{2}}\left(s, m^{2}, m^{2}\right) D_{n}\left(p_{a}, p_{b} ; p_{1} ; p_{2} ; \cdots p_{n}\right),
$$

where $\Delta(x, y, z)=x^{2}+y^{2}+z^{2}-2(x y+y z+z x)$, and each momentum $p_{i}=\left(E_{i}, p_{i}^{\prime \prime}\right)$ is specified by a rapidity variable $y_{i}, e . g$ 。 


$$
p_{a}=m\left(\operatorname{coshy}_{a}, \operatorname{sinhy}_{a}\right), p_{b}=m\left(\operatorname{coshy}_{b}, \operatorname{sinhy}_{b}\right), p_{i}^{\prime \prime} / E_{i}=\operatorname{tanhy}_{i},
$$

The energy and the longitudinal momentum sum rules can then be written as

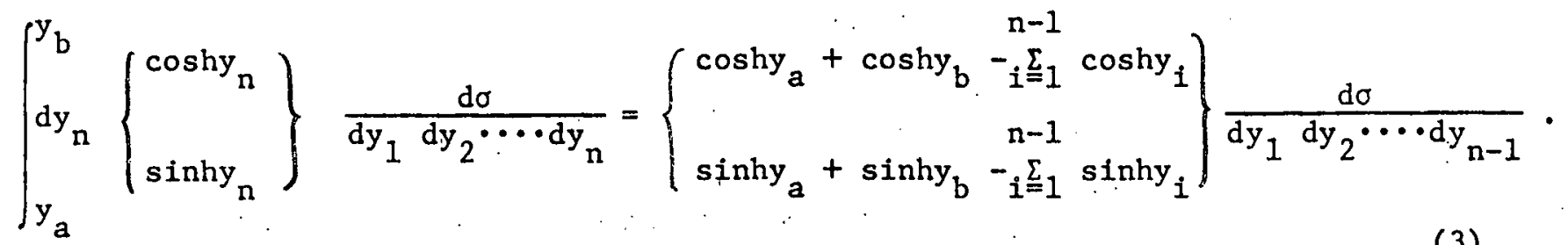

However, it is conceptually more appealing to express them in terms of discontinuities, so that they become ${ }^{7}$

$$
\begin{aligned}
& \int_{y_{a}}^{y_{b}} d y_{n} \operatorname{coshy} D_{n}\left(y_{a}, y_{b} ; y_{1}, y_{2} \cdots y_{n}\right)=\left(\operatorname{coshy} a+\operatorname{coshy} y_{b}-\sum_{i=1}^{n-1} \operatorname{coshy}_{i}\right) \\
& \times D_{n-1}\left(y_{a}, y_{b} ; y_{1}, \cdots \cdot y_{n-1}\right) \text {, } \\
& \int_{y_{a}}^{y_{b}} y_{n} \quad \operatorname{sinhy}_{n} D_{n}\left(y_{a}, y_{b} ; y_{1}, y_{2} \cdots y_{n}\right)=\left(\operatorname{sinhy}_{a}+\operatorname{sinhy}_{b}-\sum_{i=1}^{n-1} \operatorname{sinhy} y_{i}\right) \\
& x D_{n-1}\left(y_{a}, y_{b} ; y_{1} \cdots y_{n-1}\right) \text {. }
\end{aligned}
$$

Equations (4) and (5) (or Eq. (3)) are, strictly speaking, correct only in the limit $\left\langle q_{i} \phi^{2}\right\rangle \rightarrow 0$. This assumption is for notational convenience only, and, as we will point out later, it does not impair the generality of our approach. To exploit the consequences of (4) and (5), we next introduce multi-dimensional Laplace transforms for $\mathrm{D}_{\mathrm{n}} ; \mathrm{n}=0,1,2, \cdots$. 
II. Multi-Dimensional Laplace-Transform.

Lorentz invariance guarantees that $D_{n}$ on $1 y$ depends on rapidity differences; and, for $D_{n}$, there are $n+1$ independent variables. Energy-momentum conservation requires

$$
y_{a} \leq y_{i} \leq y_{b}
$$

and the complete phase space can further be divided into $n$ ! regions, each of which corresponds to a partition of the line interval $\left[y_{a}, y_{b}\right]$, characterized by

$$
y_{a} \leq y_{\lambda_{1}} \leq y_{\lambda_{2}} \leq \cdots \cdots \leq y_{\lambda_{n}} \leq y_{b}
$$

Let us first concentrate on the region $\left\{\lambda_{i}=1\right\}$. We can choose the independent variables to be

$$
\xi_{1}=y_{1}-y_{a}, \xi_{2}=y_{2}-y_{1}, \ldots ., \xi_{n}=y_{n}-y_{n-1}, \xi_{n+1}=y_{b}-y_{n},
$$

and, in this region, we denote

$$
D_{n}\left(y_{a}, y_{b} ; y_{1}, y_{2} \cdots y_{n}\right) \equiv D_{n}\left(\xi_{1}, \xi_{2}, \cdots, \xi_{n+1}\right)
$$

Since all $\xi_{1} \geq 0$, we can define a multi-Laplace transform

$$
\tilde{D}_{n}\left(\ell_{1}, \ell_{2}, \cdots, \ell_{n+1}\right) \equiv \int_{0}^{\infty} \mathrm{d} \xi_{1} e^{-\ell_{1} \xi_{1}} \ldots \int_{0}^{\infty} \mathrm{d} \xi_{n+1} e^{-\ell} e^{-\ell}{ }^{\xi_{n+1}} \mathrm{D}_{n}\left(\xi_{1}, \cdots, \xi_{n+1}\right),
$$

and the inversion formula is simply

$D_{n}\left(\xi_{1}, \cdots, \xi_{n+1}\right)=\int_{c-i \infty}^{c+i \infty} \frac{d \ell_{i}}{2 \pi i} \cdots \int_{c-1 \infty}^{c+i \infty} \frac{d l_{n+1}}{2 \pi i} e^{\ell_{1} \xi_{1}} \ldots e^{\ell_{n+1} \xi_{n+1}} \tilde{D}_{n}\left(\ell_{1}, \cdots, l_{n+1}\right)$. 
The integration contour in (11) is chosen to be to the right of all singularities of the integrand.

The complex parameter $\ell_{i}$ is essentially the complex angular momentum variable conjugate to the energy variable $\cosh \xi_{1}$. Leading asymptotic behavior of $\mathrm{D}_{\mathrm{n}}$ in $\xi_{i}$ is then controlled by the singularity of $\tilde{D}_{n}$ furthest to the right in the $\ell_{i}$-plane. In fact, leading singularities in $\ell_{i}$ are Regge singularities, and the structure differs from that of the ordinary Regge complex plane only in their "daughter" sequences. For this reason, we'refer to our $\ell$-planes as if they were the conventional J-planes. We assume that $\mathrm{D}_{n}$ 's are sufficiently well behaved at both limits so that $(10)$ is defined. This is certainly true for models we are interested in, such as the Regge pole models. 9

Although we have worked within a particular partition of $\left[y_{a}, y_{b}\right]$, the partial-wave amplitudes $\tilde{\mathrm{D}}_{\mathrm{n}}\left(\ell_{1}, \cdots, \ell_{n+1}\right)$ is actually independent of this choice because of identical particles. For models with several different species of particles; additional labels for inequivalent partitions of $\left[y_{a}, y_{b}\right]$ are also necessary for our partial-wave amplitudes. We make a further notational simplification, for $\mathrm{n}=0,1,2$, by introducing

$A(l)=\tilde{D}_{0}(l)=\int_{0}^{\infty} \mathrm{d} \xi e^{-\ell \xi} \mathrm{D}_{0}(\xi)$,

$B\left(l_{a}, \ell_{b}\right)=\tilde{D}_{1}\left(\ell_{a}, \ell_{b}\right)=\int_{0}^{\infty} \mathrm{d} \xi_{a} \int_{0}^{\infty} \mathrm{d} \xi_{b} e^{-l_{a} \xi} a e^{-\ell_{b} \xi_{b}} D_{1}\left(\xi_{a}, \xi_{b}\right)$,

$C\left(\ell_{a}, \ell_{c}, \ell_{b}\right)=\tilde{D}_{2}\left(\ell_{a}, \ell_{c}, \ell_{b}\right)=\int_{0}^{\infty} \mathrm{d} \xi_{a} \int_{0}^{\infty} \mathrm{d} \xi_{c} \cdot \int_{0}^{\infty} \mathrm{d} \xi_{b} e^{-\ell_{a} \xi_{a}} e^{-\ell_{c} \xi_{c}} e^{-\ell_{b} \xi_{b}}$

$$
\mathrm{D}_{2}\left(\xi_{\mathrm{a}}, \xi_{c}, \xi_{b}\right)
$$

\section{Diagonalization of Inclusive Sum Rules}

Before we show how Inclustve sum rules (4) and (5) can be diagonalized, we 
shall first rewrite them in a more convenient form. Multiplying the sum and the difference of (4) and (5) by $e^{-y_{a}}$ and $e^{y_{a}}$ respectively, we obtain for $\mathrm{n}=0,1, \cdots \cdots$

$$
\begin{gathered}
x_{n} \stackrel{( \pm)}{ }\left(y_{a}, y_{b} ; y_{1} \cdots ; y_{n}\right) \equiv\left(1+e^{ \pm\left(y_{b}-y_{a}\right)}-\sum_{1=1}^{n} e^{ \pm\left(y_{i}-y_{a}\right)}\right) D_{n}\left(y_{a}, y_{b} ; y_{1}, \cdots, y_{n}\right) \\
-\int_{y_{a}}^{y_{b} y_{n+1}} e^{ \pm\left(y_{n+1}-y_{a}\right)} D_{n+1}\left(y_{a}, y_{b} ; y_{1}, \cdots, y_{n+1}\right)=0
\end{gathered}
$$

This amounts to specifying rapidities in the rest frame of the particle a. More generally, we introduce relative rapidities

$$
y_{a i} \equiv y_{i}-y_{a}, y_{i j} \equiv y_{j}-y_{1}, y_{j b} \equiv y_{b}-y_{j}, y_{a b} \equiv y_{b}-y_{a}
$$

With our convention (6), we find that

$$
y_{a i} \geqslant 0, y_{j b} \geqslant 0, y_{a b} \geqslant 0
$$

and $y_{1 j}$ can be positive or negative depending on the particular, partition of $\left[y_{a}, y_{b}\right]$ in which one is working. Since there are only a few partitions, for $\mathrm{n}=1,2$, Eq. (15) can be written explicitly as

$$
x_{0}^{ \pm}\left(y_{a b}\right)=\left(1+e^{ \pm y} \cdot a b\right) D_{0}\left(y_{a b}\right)-\int_{0}^{y_{a b}} d y_{a 1} e^{+y_{a l}} D_{1}\left(y_{a 1}, y_{1 b}\right)=0,
$$

$$
\begin{gathered}
x_{1}^{( \pm)}\left(y_{a 1}, y_{1 b}\right)=\left(1+e^{ \pm y_{a b}}-e^{ \pm y_{a 1}}\right) D_{1}\left(y_{a 1}, y_{1 b}\right) \\
-\int_{0}^{y_{a l}} d y_{21} e^{ \pm y_{a 2} D_{2}\left(y_{a 2}, y_{21}, y_{1 b}\right)-\int_{0}^{y_{1 b}} d y_{12} e^{ \pm\left(y_{a 1}+y_{12}\right)}} \\
\quad D_{2}\left(y_{a 1}, y_{12}, y_{2 b}\right)=0
\end{gathered}
$$


Alternatively, we can also specify rapidities in the rest frame of the particle 1. We find that the sum rules (15), for $n=1$, can be written in a more symmetric form

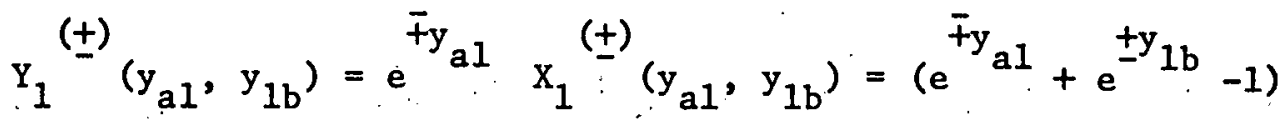

$$
\begin{aligned}
& \times D_{1}\left(y_{a 1}, y_{1 b}\right)-\int_{y_{a}}^{y_{b}} y_{2} e^{ \pm y_{21}} D_{2}=0 \text { : }
\end{aligned}
$$
(t) $\mathrm{x}_{\mathrm{n}}-\left(\mathrm{y}_{\mathrm{a}}, \mathrm{y}_{\mathrm{b}} ; \mathrm{y}_{1} \cdots \mathrm{y}_{\mathrm{n}}\right)=0, \mathrm{n}=0,1,2, \cdots$, , we need first to specify $\mathrm{a}$ particular ordering of $\left\{y_{1}\right\}$. However, for $n=0$ and 1 , there is no such freedom, Applying

$$
\int_{0}^{\infty} d y_{a b} e^{-\ell y} a b
$$

to. (18), we obtain

$$
\begin{aligned}
& \tilde{x}_{0}^{( \pm)}(l)=\int_{a b}^{\infty} d^{-\ell y_{a b}} x_{0}^{( \pm)}\left(y_{a b}\right) \\
& \quad=A(l)+A(l \mp 1)-\int_{0}^{\infty} d y_{a b} e^{-\ell y_{a b}} \cdot \int_{0}^{y_{a b}} d y_{a l} e^{ \pm y_{a l}} D_{1}\left(y_{a l}, y_{1 b}\right)=0 .
\end{aligned}
$$

Interchanging the order of $\mathrm{y}_{\mathrm{ab}}, \mathrm{y}_{\mathrm{al}}$ integrations, and changing the integration variable from $y_{a b}$ to $y_{1 b}=y_{a b}-y_{a l}$, we arrive at the following equations,

$x_{0}(l)=A(l)+A(l \mp 1)-B(l \mp 1, l)=0$

Similarly, applying

$$
\int_{0}^{\infty} d y_{a l} e^{-\ell a y} a \cdot 1 \int_{0}^{\infty} d y_{1 b} e^{-\ell b y} 1 b
$$


$-8-$

to Eq. (19), we obtain

$$
\begin{aligned}
\tilde{x}_{1}^{( \pm)}\left(l_{a}, \ell_{b}\right)= & B\left(l_{a}, l_{b}\right)+B\left(l_{a} \mp 1, \ell_{b} \mp 1\right)-B\left(l_{a} \mp 1, \ell_{b}\right) \\
& -C\left(l_{a} \mp 1, l_{a}, \ell_{b}\right)-C\left(l_{a} \mp 1, \ell_{b} \mp 1, l_{b}\right)=0
\end{aligned}
$$

Alternatively, if $(20)$ is used, we have

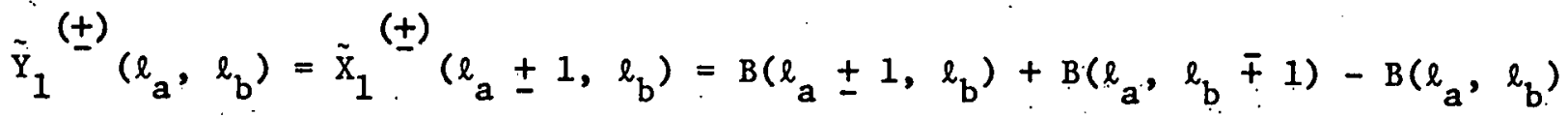

$$
\begin{aligned}
& -C\left(\ell_{a}, \ell_{a} \pm 1, \ell_{b}\right)-C\left(\ell_{a} ; \ell_{b} \mp 1, \ell_{b}\right)=0
\end{aligned}
$$

In a analogous manner, we can obtain, for general $n$,

$$
\tilde{x}_{n}^{(+)}\left(l_{1}, \ldots, \ell_{n+1}\right)=0 \text {, }
$$

relating $\tilde{D}_{n}$ to $\tilde{D}_{n+1}$. We shall not write this general form here.

We can now remove our unphysical restriction on transverse momenta of all produced particles. We simply carry out the same analysis with $\vec{q}_{\perp} \downarrow$ kept as an additional label. In terms of normalizations for $D_{n}\left(p_{a}, p_{b} ; p_{i} \cdots p_{n}\right)$, used in Ref. 6 , we find that, for $n=0$ and $n=1$,

$$
\begin{aligned}
& \tilde{\mathrm{D}}_{0}(l)+\tilde{\mathrm{D}}_{0}(l+1)-\left[(2)(2 \pi)^{3}\right]^{-1} \int \mathrm{d}_{1}^{\perp}\left(1+\overrightarrow{\mathrm{q}}_{1}^{\perp} / \mathrm{m}^{2}\right)^{\frac{1}{2}} \tilde{\mathrm{D}}_{1}\left(l \mp 1, \overrightarrow{\mathrm{q}}_{1}^{\perp 2}, l\right)=0, \\
& \tilde{\mathrm{D}}_{1}\left(\ell_{a} \pm 1, \overrightarrow{\mathrm{q}}_{1}^{+}, \ell_{\mathrm{b}}\right)+\tilde{\mathrm{D}}_{1}\left(l_{a}, \overrightarrow{\mathrm{q}}_{1}^{\frac{1}{2}}, \ell_{b} \mp 1\right)-\left(1+\overrightarrow{\mathrm{q}}_{1}^{\perp^{2}} / \mathrm{m}^{2}\right)^{\frac{1}{2}} \tilde{\mathrm{D}}_{1}\left(\ell_{a}, \overrightarrow{\mathrm{q}}_{1}^{\frac{1}{2}}, \ell_{b}\right) \\
& -\left[(2)(2 \pi)^{3}\right]^{-1} \int d \vec{q}_{2}\left(1+\vec{q}_{2}^{+2} / m^{2}\right)^{\frac{1}{2}}\left[\tilde{D}_{2}\left(\ell, \vec{q}_{2}^{1}, \ell a \pm 1, \vec{q}_{1}^{+} ; \ell_{b}\right)\right. \\
& \left.+\tilde{\mathrm{p}}_{2}\left(\ell_{\mathrm{a}}, \overrightarrow{\mathrm{q}}_{1}^{\perp}, \ell_{b} \mp 1, \vec{q}_{2}^{\perp}, \ell_{b}\right)\right]=0
\end{aligned}
$$


If $D_{n}$ 's are strongly damped in $\left|\vec{q}_{1}^{\alpha}\right|$, then (24) and (25) can easily be reduced to (22) and (23)'. In general, ${ }^{10}$ a Fourier analysis in the transverse angle $\phi_{12}, \cos \phi_{12} \equiv \hat{q}_{1}^{\perp} \cdot \hat{q}_{2}^{+}$, can be made for $\tilde{D}_{2}$, so that each Fourter component will depend only on the magnitudes $\left|\vec{q}_{1}\right|$ and $\left|\vec{q}_{2}^{1}\right|$. However, for our subsequent discussions, we shall return to Eqs. (22) and (23)'.

IV. Bootstrap Models

The necessary assumptions for the existence of our multi-dimensional Laplace transforms immediately imply that $\tilde{D}_{n}\left(l_{1}, \because,, l_{n+1}\right)$ vanishes in the $11 m 1 t \operatorname{Re}\left(l_{1}\right) \rightarrow \infty$, so that they can be represented by a dispersion integral, e.g.

$$
A(l)=\frac{1}{2 \pi 1} \int_{c-1 \infty}^{c+1 \infty} d l^{\prime} \frac{A\left(\ell^{\prime}\right)}{\ell-\ell^{\prime}}
$$

The integration contour can be deformed to the left, picking up singularitles of $A\left(l^{\prime}\right)$ along the way. Let us assume that there exists several poles at $\ell=\alpha_{i}$, $\operatorname{Re}\left(\alpha_{i}\right)>\operatorname{Re}\left(\alpha_{j}\right)$, for $i<j, i, j=0,1, \cdots, m$. We can then choose $c^{\prime}<\operatorname{Re}\left(\alpha_{m}\right)$, and represent $A(l)$ by

$$
A(l)=\sum_{i=0}^{m} \frac{G_{i}^{2}}{l-\alpha_{1}}+\frac{1}{2 \pi i} \int_{c^{\prime}-1 \infty}^{c^{\prime}+i \infty} \mathrm{d} \ell^{\prime} \frac{A\left(l^{\prime}\right)}{\ell-\ell^{\prime}},
$$

where $G_{i}{ }^{2}$ is the residue of $A(l)$ at $\ell=\alpha_{1}$. It follows from (22) that $B(l, \ell+1)$ also have poles at $l=\alpha_{1}$, and are analytic for $\operatorname{Re}(l)>\alpha_{0}$. Assuming that $B\left(\ell_{a}, l_{b}\right)$ does not have singularities in $\ell_{a}\left(l_{b}\right)$ that are $\ell_{b}\left(l_{a}\right)$ dependent in the region $\operatorname{Re}\left(l_{a}\right)>c^{\prime}\left(\operatorname{Re}\left(l_{b}\right)>c^{\prime}\right)$, we can then conclude that $B\left(l_{a}, l_{b}\right)$ has poles at $\ell_{a}=\alpha_{i}$ and $\ell_{b}=\alpha_{j}$, and it can be represented by 


$$
\begin{aligned}
& B\left(l_{a}, \ell_{b}\right)=\sum_{i=0}^{m} \frac{\gamma_{1}}{l_{a}-\alpha_{i}}+\sum_{i=0}^{m} \frac{\gamma_{i}}{l_{b}-\alpha_{1}}+\sum_{i, j=0}^{m} \frac{\beta_{1 j}}{\left(l_{a}-\alpha_{i}\right)\left(l_{b}-\alpha_{j}\right)} \\
& +\int_{c^{\prime}-1 \infty}^{c !^{\prime}+1 \infty} \frac{d \ell^{\prime} a}{2 \pi i} \int_{c^{\prime}-i \infty}^{c^{\prime}+i \infty} \frac{d l^{\prime}}{2 \pi i} \cdot \frac{B\left(l_{a}^{\prime}, \ell_{b}{ }^{\prime}\right)}{\left(l_{a}-l_{a}^{\prime}\right)\left(\ell_{b}-\ell_{b}{ }^{\prime}\right)} \\
& =\sum_{i=0}^{m} \frac{G_{i} G_{i}^{0}}{l_{a}-\alpha_{i}}+\sum_{i=0}^{m} \frac{G_{i}{ }^{0} G_{i}}{l_{b}-\alpha_{i}} \\
& +\sum_{1, j=0}^{\infty} G_{i} \frac{1}{l_{a}-\alpha_{1}} g_{i j} \frac{1}{\ell_{b}-\alpha_{j}} \ddot{G}_{j}+\text { (Background integral). }
\end{aligned}
$$

Performing the inversion procedure, (11); țogether with Eq. (1), we find that inclusive cross sections now have the desired scaling properties. In fact, what we have outlined is precisely the Regge assumption made by Mueller. ${ }^{11}$ We stress that the "independence" of singularities of $B\left(\ell_{a}, l_{b}\right)$ is a debatable assumption. It holds in dynamical models discussed in Ref. 11, but certalnly not true for the fragmentation model of Yang and his collaborators. 12 It is not our present purpose to demonstrate that Regge behavior follows from our sum rules, but rather to obtain restrictions on Regge couplings due to unitarity constraints. Therefore, the usual Regge expansion is a dynamical input for us. Together with the "independence" of singularities assumption, we also postulate the factorization of Regge residues. $13^{\circ}$ Applying these two assumptions to " $C\left(\ell_{a}, \ell_{c}, \ell_{b}\right)$; we find.

$$
\begin{aligned}
C\left(l_{a}, l_{c}, l_{b}\right)= & \int_{1, j, k=0}^{m} G_{a} \frac{1}{\ell_{a}-\alpha_{i}} g_{i j} \frac{1}{\ell_{c}-\alpha_{j}} g_{j k} \frac{1}{\ell_{b}-\alpha_{k}} G_{k} \\
& +(\text { (Double-pole terms) }+ \text { (Single-pole terms) } \\
& + \text { (Background integral) }
\end{aligned}
$$


In Eq. (28), the first single pole term on the right hand side only contributes to the limiting distribution of $\mathrm{b}$, the second term contributes to the limiting distribution of a, the third term contributes to both, and the background integral contribution is asymptotically negligible. Therefore, to have "asymptotic" consistency, we need only to equate residues at

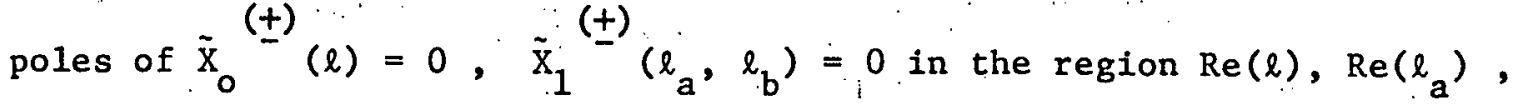
$\operatorname{Re}\left(\ell_{b}\right)>c^{\prime}$. This automatically' leads to non-linear relations among $g_{i j}$, $G_{j}, G_{k}{ }^{0}, \alpha_{i}$, etc. To illustrate the mathematical structure of the resulting equations, we assume that the background term as well as the single pole term in $B$ and the single and the double pole terms in $C$ can be ignored. This is not completely unrealistic if we keep enough Regge poles. We first evaluate $\tilde{\mathrm{X}}_{0}{ }^{(t)}(\ell)=0$ at $\ell=\alpha_{i}+1,\left(\tilde{\mathrm{X}}_{0}{ }^{(-)}(\ell)=0\right.$ does not yield any new information because of the symmetry of $\left.B\left(l_{a}, \ell_{b}\right)\right)$, and find

$$
G_{i}=\sum_{j=0}^{m} \frac{g_{i j} G_{j}}{\alpha_{i}-\alpha_{j}+1}
$$

This "eigenvalue" condition guarantees the "asymptotic" consistency 14 of Eq. (18). The same minimal asymptotic consistency for $\mathrm{Eq}$. (19). requires the vanishing of residues of $\tilde{Y}_{1}{ }^{(+)}\left(l_{a}, \ell_{b}\right)=0 \cdot\left(\tilde{Y}_{1}{ }^{(-)}\left(l_{a}, \ell_{b}\right)=0\right)$ at $\ell_{a}=\alpha_{i}\left(l_{a}=\alpha_{1}+1\right)$, and $\ell_{b}=\alpha_{j}+1\left(\ell_{b}=\alpha_{j}\right)$. It is interesting that these conditions are automatically satisfied if Eq. (30) holds.

Very stringent conditions result if we demand further consistency at lower poles. Under the same simplifying assumptions and assuming $\alpha_{i}-\alpha_{j} \neq \pm 1$, we find that $\tilde{x}_{0}^{(+)}\left(\ell=\alpha_{i}\right)=0$ implies

$$
G_{i}=\sum_{j=0}^{m} \frac{g_{i j} G_{j}}{\alpha_{1}-\alpha_{j}-1}
$$


and $\tilde{Y}_{1}^{( \pm)}\left(\ell_{a}=\alpha_{1}, l_{b}=\alpha_{j}\right)=0$ imply

$$
g_{i j}=g_{j i}=\sum_{k=0}^{m} \frac{\left(\alpha_{i}+\alpha_{j}-2 \alpha_{k}\right)}{\left(\alpha_{i}-\alpha_{k}+1\right)\left(\alpha_{k}-\alpha_{j}+1\right)} g_{i k} g_{k j}
$$

These are highly non-linear constraints on Regge couplings. Due to our numerous unphysical assumptions, we do not attempt to solve these equations here. It is interesting to note that Eqs. (30)-(32) are not compatible with each other if only one or two poles are kept. 15

\section{Additional Remarks}

We would like to comment further on our approximate dynamical scheme of Section IV. First, branch point contributions should in general be included in our expansions (27)-(29). Although their inclusion will not cause any difficulty of principles, we no longer may invoke the factorization assumption. It is therefore unlikely for us to achieve a complete bootstrap including both poles and branch points. However, if their effects are used as additional inputs, ${ }^{16}$ partial bootstraps can result. Second, we discuss the effect of finite truncation in Regge singularities. There is indeed a chance of realism if the discontinuity $D_{n}$ always remains finite (it may diverge) when relative rapidities $y_{i j}, y_{a i}, y_{j b} \rightarrow 0$, $i_{0} e_{0}$, this behavior can be simulated by a finite sum of Regge pole terms. However, if $D_{n}$ diverges, our approximations $(27)(29)$ are certainly inadequate. One possible way out is to keep the singlepole term in (27) and to choose $G^{0}$ in such a way to represent the average effect of this divergent behavior ${ }^{17}$ (such as triple-Regge behavior with $2 \alpha(t)-\alpha(0)>0)$.

We mention in passing that one of the requirements for the existence of our multi-dimensional Laplace transforms is that $D_{n}^{\prime}$ 's can not be too singular 
as $y_{i b}, y_{a i} \rightarrow 0, e . g .$, if $y_{a 1} i .0, D_{n} \propto\left(1-e^{-y_{a l}}\right)^{-\varepsilon}$, we require, $\varepsilon<1$. Since $D_{0}<\infty$. when $y_{a b} \rightarrow 0, A(l)$ is therefore well-defined. However, if $\alpha(0)=1$, it follows that $D_{1}\left(y_{a 1}, y_{1 b}\right) \alpha\left(1-e^{-y_{a 1}}\right)^{-1}$ as $y_{a 1} \rightarrow 0$. Our sum rule $\tilde{\mathrm{x}}_{0} \stackrel{( \pm)}{ } \cdot(l)=0$ then forces the triple-Pomeranchon coupling to vanish in the forward limit because $B(l, \ell \pm 1$ ) has to be finite (1.e., it is equal to $A(l)+A(l \pm 1))$. This is precisely equivalent to the argument given in $\operatorname{Ref.} 2$, but now in the language of the partial-wave analysis. One possible advantage of this present approach is our being able to see explicitly that other singularities, such as branch points at $J=2 \alpha(0)-1$, can not change this conclusion because they come from divergences at the upper limit of $\xi_{\text {a }}$ integration in (13).

We are fully aware that simple models without incorporating internal symmetry, baryon number, etc. are of very little real use. It is perhaps a blessing that a single- and a two-pole models are not compatible with our unitarity constraints, The inclusion of isospin, for instance, increases not only our internal trajectory multiplicity but also external particle species. This added structure may allow our sum rules to sustain a realistic bootstrap system. Results of investigations along thị line will be reported elsewhere.

Despite its shortcomings, our bootstrap model of section IV does have many interesting and desirable features. Since Eq. (30) is not too difficult to satisfy, it follows that the two-particle correlation function ${ }^{18}$ exhibits the features of short-range correlation in the relative rapidities. We find that

$$
\begin{aligned}
C_{12}\left(y_{a}, y_{1}, y_{2}, y_{b}\right) \equiv D_{0}\left(y_{a b}\right) & -1 \\
& -D_{1}\left(y_{a}\left(y_{a}, y_{1}, y_{1 b}, y_{b}\right)\right. \\
& \left.D_{1}\left(y_{a 2}, y_{2 b}\right) D_{0}^{-1}\left(y_{a b}\right)\right\}
\end{aligned}
$$

in general is non-zero whenever any' one of the relative rapidities $y_{a i}, y_{i j}, y_{j b}$ is not infinite. Inclusive sum rules, therefore, do not directly infer any 
constraints on the value of correlation length: 19

Our multi-dimensional. Laplace transforms can also be used to study other sum rules of the type $2-4$

$$
\begin{aligned}
& \int d p \frac{d \sigma}{d p}=\left\langle n>\sigma^{t o t}\right. \\
& \int d p_{1} d p_{2} \frac{d \sigma}{d p_{1} d p_{2}}=\left\langle n(n-1)>\sigma^{t o t}\right.
\end{aligned}
$$

It follows from $(12) \div(14)$ that

$$
\begin{gathered}
<n>n\left(\sigma^{t o t}\right)^{-1} \int_{c-i \infty}^{c+i \infty} \frac{d \ell}{2 \pi i} e^{(l-1) y_{a b}} B(l, l) \sim(\text { const }) \text { lns } \rightarrow \infty \\
<n(n-1)>\sim 2\left(\sigma^{t o t}\right)^{-1} \int_{c-1 \infty}^{c+i \infty} \frac{d l}{2 \pi i} e^{(l-1) y_{a b}} c(l, l, l) \sim(\text { const })(l n s)^{2} \rightarrow \infty,
\end{gathered}
$$

and

$$
\langle n(n-1)\rangle-\langle n\rangle^{2} \sim \text { (const) lns } \rightarrow \infty,
$$

with proportionality constants directly related to residues of poles of our partial-wave amplitudes. ${ }^{20}$. Conversely, experimental measurements of these average quantities can yield informations on asymptotic behavior of inclusive cross sections through Eq。(11).

\section{Acknowledgement}

We are grateful to R. Brower, A. DiGiacomo, H. M. Fried, K. Kang, H. Tye and G. Veneziano for discussions. 
Footnotes and References

1. G. Veneziano, Phys. Rev. Letters 28, 578 (1972); Phys. Letters 36B, 397 (1971).

2. T. T. Chou, and C. N. Yang, Phys. Rev. Letters 25, 1072 (1971).

3. C. DeTar, D. Freedman, and G. Veneziano, Phys. Rev. D4, 906 (1971).

4. K. H. Biebl and J. Wolf, Phys. Letters 37B, 197 (1971); E. Predazz1 and G. Veneziano, Nuovo Cimento Letters 2 , 749 (1971); S. H.-H. Tye (MIT Report No. CTP 239); L. S. Brown, Phys. Rev. D5, 748 (1972).

5. Cahill and Stapp, Berkeley Report No. LBL-381, 1971 (to be published).

6. C.-I Tạn, "Construction of Multiperipheral Dynamical Equations and. Inclusive Sum Rules" (to be published in Phys. Rev.). A factor of $(2 \pi)^{+3 n}$ was inadvertently left out on the left-hand side of $\mathrm{Eq} .(2.1)$ there.

7. We have left out the inhomogeneous terms to simplify the writings. For the dynamical models discussed in Section IV, we believe they are not important. For their dynamical effects in a different context, see Ref. 6 .

8. Questions relating to the triple-Regge behavior are discussed in Section $V$. In particular, it emerges naturally from our analysis that the triplePomeranchon coupling $g(t)$ vanishes at $t=0$ if $\alpha(t=0)=1$.

9. Generally, we need the assumption of polynomial boundedness in $\left|\cosh \xi_{i}\right|$.

10. Details concerning the diagonalization of sum rules with transverse momenta will be discussed elsewhere.

11. A. Mueller, Phys. Rev. D2, 2962 (1970); D. Silverman and C.-I Tan, Phys. Rev. D3, 991 (1971), and references therein.

12. J. Benecke, T. T. Chou, C. N. Yang, and E. Yen, Phys. Rev. 188, 2159 (1969).

13. Because of the $\cos \phi_{i j}$ dependence, daughter poles are in general degenerate. For our present discussion, we shall ignore the presence of daughters, i.e., 
we assume $\quad \alpha_{i}-\alpha_{j} \neq \pm 1$.

14. This level of asymptotic consistency has also been discussed by H. Tye and G. Veneziano, Phys. Letters 38B, 30 (1972); and by L. Caneschi, Phys. Letters 37B, 288 (1971). They did not, however, explicitly write down this eigenvalue condition. At this same level, T. C. Yang (La Jolla preprint) has attempted to derive a lower bound on the separation between the leading two Regge trajectories. Unfortunately, his result, as is evident from our analysis, is incorrect. (See further discussion on the two-particle correlation function in Section $v_{.}$)

15. This conclusion has also been reached independently in a direct calculation of the sum rules by A. DiGiacomo (private communication).

16. Such as the use of branch cuts in the multiperipheral models.

17. It is easy to see that threshold behavior of $D_{n}$ corresponds to asymptotic behavior of $\tilde{\mathrm{D}}_{n}\left(l_{i}\right)$ as $\operatorname{Re}\left(\dot{l}_{i}\right)++\infty$. A more definite procedure, therefore, is to perform the appropriate subtraction at $\operatorname{Re}\left(\ell_{1}\right)=+\infty$.

18. See, for instance, K. Wilson, CLNS-131, 1970 (unpublished); Jen, Kang, Shen, and Tan, Phys. Letters $38 \mathrm{~B}, 81$ (1972). It is also clear that $\mathrm{C}$ is a useful concept only in the central region where one is free of kinematic effects.

19. That is, inclusive sum rules, at the minimal consistency level, Eq. (30), are sufficiently flexible so as not to restrict the value of $\alpha_{0}-\alpha_{1}$, $\alpha_{0}$, $\alpha_{1}$ being the two leading Regge poles. This is not to be confused, however, with the more stringent requirements Eqs. (31) and (32).

20. C. Quigg, J.-M. Wang, and C. N. Yang (Stony Brook preprint). Based on their intuitive fragmentation picture, they have speculated that

$$
\langle n(n-1)\rangle-\langle n\rangle^{2} \sim s^{\alpha} \rightarrow \infty
$$


with $\alpha \leq 1 / 2$. For $\alpha \neq 0$, this prediction is very different from ours at infinite energy. However, a mere increase in this difference with energy at present machine energies cannot be used to support their picture. We note that the coefficient of the lns term in Eq. (38) has been shown to be "positive" in multiperipheral models where the correlation is provided by the Regge cut. See: S. Y. Mak and C.-I Tan, "Correlation Lengths, Inclusive Transverse Momentum Distributions and Multiperipheralism", Brown University Preprint No. C00-3130TA-257. The observation made by Yang, et. al. has also been noted before by L. Caneschi, Ref. TH. 1419-CERN, Oct. (1971). 